\title{
Achieving Anomalous Refraction with Metasurfaces Composed by Two Ordinary Dielectric Materials
}

\author{
$\underline{\text { N. L. Tsitsas }}^{1}$ and C. A. Valagiannopoulos ${ }^{2}$ \\ ${ }^{1}$ Aristotle University of Thessaloniki, Department of Informatics, GR-54124, Thessaloniki, Greece \\ ${ }^{2}$ Nazarbayev University, Department of Physics, KZ-010000, Astana, Kazakhstan \\ ntsitsas@csd.auth.gr
}

\begin{abstract}
The optimal parameters of a dielectric metasurface, composed of two alternating rectangular rods, are investigated so that it exhibits significant enhancement in the -1 diffracted order in the transmission region. An efficient integral-equation methodology is used for the numerical computations. Representative results of initial optimizations are presented.
\end{abstract}

\section{INTRODUCTION}

Negative refraction is the key mechanism behind various interesting phenomena related to the efficient manipulation of electromagnetic waves [1]. These effects include overcoming the diffraction limit of conventional lenses [2], obtaining subwavelength imaging in photonic crystals [3], and synthesizing and controlling the sign of dispersion [4]. Several metamaterial configurations, characterized by significant negative refraction, have been proposed; indicatively, we refer to semiconductor metamaterials [5], stacked plasmonic waveguides [6], parity-time symmetric metasurfaces [7], and isotropic metamaterial Huygens' surfaces [8].

In [9] and [10], it was demonstrated that a gradient dielectric metasurface composed of two periodically alternating lossless materials can generate anomalous reflection phenomena in the visible frequency range; namely the power of the -1-reflected order is significantly enhanced while the corresponding powers of the 0-reflected and transmitted orders (dictated by Snell's law) are annihilated. The effective properties of the metasurface's unit cell offering these characteristics can be found in ordinary dielectrics. The width and period of the metasurface were systematically optimized by applying a highly efficient integral-equation methodology, developed in [11] and [12].

In this work, we investigate the corresponding anomalous transmission phenomena in which the main contribution in the transmission region comes from the -1-transmitted order. Hence, an optimized gradient dielectric metasurface possessing this property can actually generate negative refraction in free space. Some initial optimization results are reported concerning the geometrical and physical parameters of realizable dielectric metasurfaces exhibiting significantly enhanced power in the -1 -transmitted order.

\section{Mathematical Analysis of the Scattering Problem}

The geometrical configuration of the examined two-dimensional gradient dielectric metasurface is depicted in Fig. 1. It comprises a $\Lambda$-periodic slab of thickness $w$ composed (per unit cell) of two lossless dielectric materials with refractive indices $n_{1}$ and $n_{2}$, respectively. The duty cycle of the material with refractive index $n_{2}$ is denoted by $s$. The entire structure lies in vacuum with refractive index $n_{0}=1$. A TE-polarized incident plane wave impinges on the metasurface at an angle of incidence $\theta$. The total electric field induced in every region is $y$-polarized, i.e. $\mathbf{E}(x, z)=\Psi(x, z) \hat{\mathbf{y}}$, and the unknown electric-field factor $\Psi(x, z)$ admits the integral representation [11]-[13]

$$
\Psi(x, z)=\Psi_{0}(x, z)+k_{0}^{2}\left(n_{2}^{2}-n_{1}^{2}\right) \iint_{S} G\left(x, z ; x^{\prime}, z^{\prime}\right) \Psi\left(x^{\prime}, z^{\prime}\right) \mathrm{d} x^{\prime} \mathrm{d} z^{\prime}, \quad(x, z) \in \mathbb{R}^{2},
$$

where $S$ denotes the total transverse cross-section of the inclusions with refractive index $n_{2}$ and $k_{0}=2 \pi / \lambda_{0}$ the vacuum wavenumber (with $\lambda_{0}$ the vacuum wavelength). Functions $\Psi_{0}$ and $G$ are the electric-field factor and the Green's function both induced on the homogeneous dielectric slab of refractive index $n_{1}$ due to the plane incident wave and a line-source excitation inside the slab, respectively. 


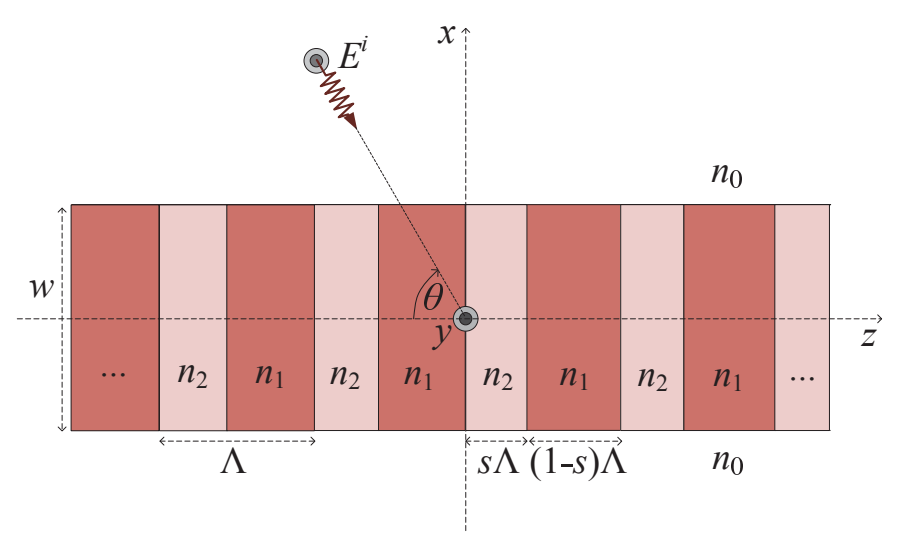

Fig. 1: Geometrical configuration of the examined gradient dielectric metasurface and the incident field.

Eq. (1) is reduced to an integral equation with respect to $u(x, z)=\exp \left(i k_{0} \cos \theta z\right) \Psi(x, z)$, which is a $\Lambda$ periodic function of $z$. Then, we apply a highly-efficient entire-domain Galerkin technique, as described in [11] and [12], and obtain a finite linear system for the coefficients of the Fourier series of $u(x, z)$ on the inclusions domain. In this way, we get the expressions of the electric field factors in the reflection and transmission regions

$$
\Psi^{r}(x, z)=\sum_{p=-\infty}^{+\infty} r_{p} \exp \left[i\left(k_{x, p} x-k_{z, p} z\right)\right], \Psi^{t}(x, z)=\sum_{p=-\infty}^{+\infty} t_{p} \exp \left[-i\left(k_{x, p} x+k_{z, p} z\right)\right],
$$

where $r_{p}$ and $t_{p}$ denote the determined complex coefficients of the $p$-th diffracted order, and $k_{x, p}$ and $k_{z, p}$ the components of the reflected and transmitted wavevectors.

The 0 -order fields are dictated by Snell's law and are always propagating. For $p \neq 0$, we define the thresholds

$$
p^{ \pm}= \pm\left(\Lambda / \lambda_{0}\right)(1 \mp \cos \theta)
$$

and see that for $p^{-}<p<p^{+}$, the $p$-order reflected and transmitted fields are propagating along $x$, while for $p>p^{+}$or $p<p^{-}$, the $p$-order fields are evanescent.

\section{Optimization Procedure AND Numerical Results}

We consider $p^{+}<1$ and $-2<p^{-}<-1$ in order to assure that only the 0 - and the -1 -reflection and transmission orders propagate. Thus, we obtain the following condition

$$
\max \{1-\cos \theta,(1+\cos \theta) / 2\}<\lambda_{0} / \Lambda<1+\cos \theta .
$$

Then, we proceed by following a similar but more general optimization procedure to that of [9], namely first we fix $\theta=60^{\circ}$ and obtain by (4) the interval of variation of the period $\Lambda$ for a chosen wavelength $\lambda_{0}$. For the refractive indices $n_{1}$ and $n_{2}$ of the two constituent materials, we have examined several combinations of realizable lossless materials in the visible frequency range. Next, we follow a systematic optimization scheme to determine the optimal values of the thickness $w$, duty cycle $s$, and period $\Lambda$ offering significantly enhanced powers in the -1transmission order with respect to the powers of the -1 - reflection and 0- reflection and transmission orders; the power of the $p$-th diffracted order in the reflection and transmission region is, respectively, defined by

$$
P_{p}^{r}=\frac{\left|r_{p}\right|^{2}}{\sin \theta} \sqrt{1-\left(\cos \theta+p \frac{\lambda_{0}}{\Lambda}\right)^{2}}, P_{p}^{t}=\frac{\left|t_{p}\right|^{2}}{\sin \theta} \sqrt{1-\left(\cos \theta+p \frac{\lambda_{0}}{\Lambda}\right)^{2}} .
$$

Some representative results of initial optimizations are depicted in Fig. 2. These concern the variations of $P_{0}^{r}, P_{0}^{t}, P_{-1}^{r}$, and $P_{-1}^{t}$ versus the period $\Lambda$ of the metasurface for the optimally determined values of the thickness $w$ and duty cycle $s$. We consider that $n_{1} \cong 1.35$ (which can be realized e.g. by Teflon AF fluoropolymers), while $n_{2}$ is assumed to obey the frequency-varying model of lossless Silicon. The maximum attained values of $P_{-1}^{t}$ are $96 \%$ and $97 \%$ for the blue and green color, respectively, while the metasurface's parameters are $w=85 \mathrm{~nm}$, $\Lambda=457 \mathrm{~nm}, s=0.5$ (for the blue color) and $w=105 \mathrm{~nm}, \Lambda=546 \mathrm{~nm}, s=0.5$ (for the green color). 

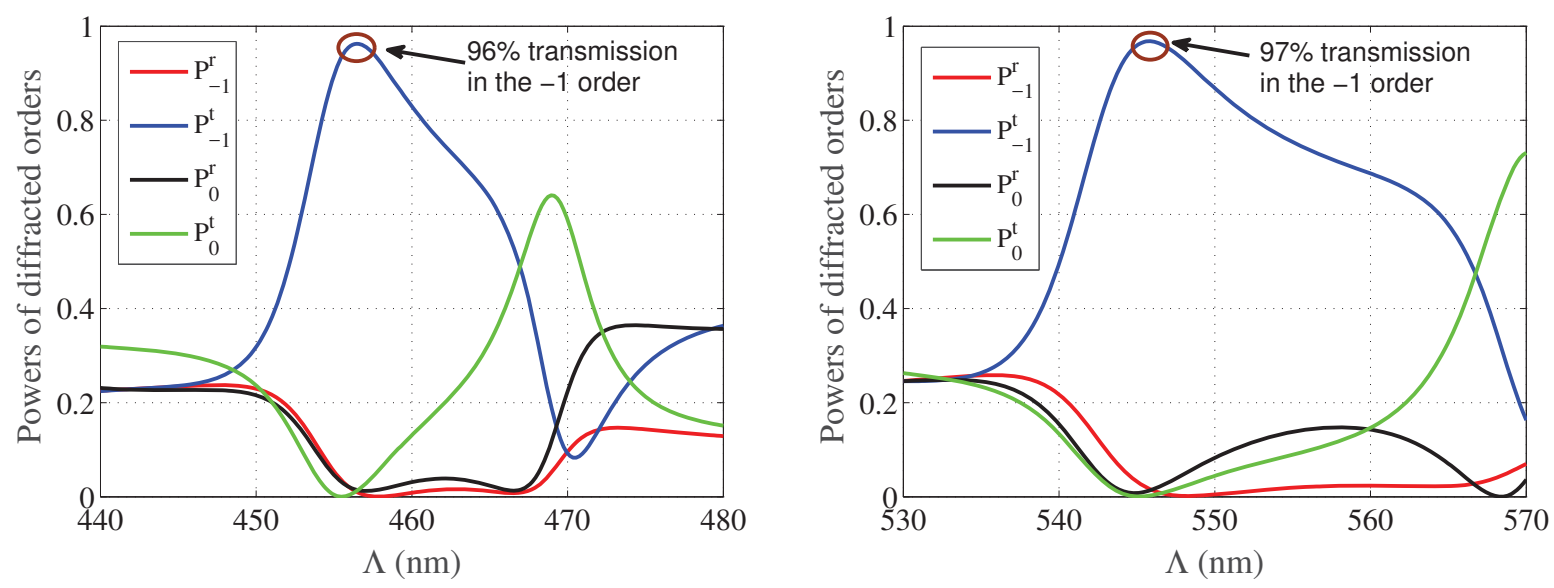

Fig. 2: Diffracted powers versus the period $\Lambda$ for (a) $\lambda_{0}=470 \mathrm{~nm}$ (blue color) and (b) $\lambda_{0}=532 \mathrm{~nm}$ (green color).

\section{CONCLUSIONS}

A rigorous integral equation methodology was used as a fast and efficient optimization platform for determining the optimal parameters of a metasurface achieving nearly total transmission in the -1 -order. Results of some initial optimizations were depicted. Additional results have been computed and will be presented at the conference.

\section{ACKNOWLEDGEMENT}

This work was partially supported by Nazarbayev University Small Grants with project entitled: "Super transmitters, radiators and lenses via photonic synthetic matter" (no. 17095). Funding from MES RK state-targeted program BR05236454 is also acknowledged.

\section{REFERENCES}

[1] G.V. Eleftheriades and K.G. Balmain (Eds.), Negative Refraction Metamaterials, Fundamental Principles and Applications, New Jersey: IEEE Press, 2005.

[2] J.B. Pendry, "Negative Refraction Makes a Perfect Lens," Physical Review Letters, vol. 85, pp. 3966-3969, 2000

[3] C. Luo, S.G. Johnson, J.D. Joannopoulos, and J.B. Pendry, "All-angle negative refraction without negative effective index," Physical Review B, vol. 65, 201104(R), 2002.

[4] F. Siddiqui, M. Mojahedi, and G.V. Eleftheriades, "Periodically loaded transmission line with effective negative refractive index and negative group velocity," IEEE Transactions on Antennas and Propagation, vol. 51, pp. 2619-2625, 2003.

[5] A.J. Hoffman, L. Alekseyev, S.S. Howard, K.J. Franz, D. Wasserman, V.A. Podolskiy, E.E. Narimanov, D.L. Sivco, and C. Gmachl, "Negative refraction in semiconductor metamaterials," Nature Materials, vol. 6, pp. 946-950, 2007.

[6] T. Xu, A. Agrawal, M. Abashin, K.J. Chau, and H.J. Lezec, "All-angle negative refraction and active flat lensing of ultraviolet light," Nature, vol. 497, pp. 470-474, 2013.

[7] R. Fleury, D.L. Sounas, and A. Alù, "Negative Refraction and Planar Focusing Based on Parity-Time Symmetric Metasurfaces," Physical Review Letters, vol. 113, 023903, 2014.

[8] C. Pfeiffer, N.K. Emani, A.M. Shaltout, A. Boltasseva, V.M. Shalaev, and A. Grbic, "Efficient Light Bending with Isotropic Metamaterial Huygens' Surfaces," Nano Letters, vol. 14, pp. 2491-2497, 2014.

[9] N.L. Tsitsas and C.A. Valagiannopoulos, "Anomalous reflection of visible light by all-dielectric gradient metasurfaces," Journal of the Optical Society of America B, vol. 34, pp. D1-D8, 2017.

[10] N.L. Tsitsas and C.A. Valagiannopoulos, "Mitigating Snell's-Law Reflection and Transmission with Metasurfaces of Ordinary Dielectrics," Proceedings of ICEAA 2017, pp. 1160-1163, Verona, Italy, 11-15 September 2017.

[11] N.L. Tsitsas, N.K. Uzunoglu, and D.I. Kaklamani, "Diffraction of plane waves incident on a grated dielectric slab: An entire domain integral equation analysis," Radio Science, vol. 42, RS6S22, 2007.

[12] N.L. Tsitsas, "Efficient integral equation modeling of scattering by a gradient dielectric metasurface," EPJ Applied Metamaterials, vol. 4, 3, 2017.

[13] N. L. Tsitsas, D. I. Kaklamani, and N. K. Uzunoglu, "Rigorous integral equation analysis of nonsymmetric coupled grating slab waveguides," Journal of the Optical Society of America A, vol. 23, pp. 2888-2905, 2006. 